\section{FEEDBACK AMPLIFIERS}

\author{
By C. A. A. WASS
}

$\mathrm{T}$ HE use of negative feedback to give improved performance in a number of important respects is now a well-established feature of amplifier design technique, and the simple mathematical treatment of an amplifier with feedback has been given by a number of writers. The earliest of these accounts was in a paper by Black ${ }^{1}$, who showed that if an amplifier has a voltage gain of $\mu$ times, and an attenuating network is connected between the output terminals and the input terminals in such a way that a voltage equal to a fraction $\beta$ of the output voltage is connected in series with, and in opposite phase to, the input voltage, the amplifier gain will be reduced in the ratio $1 /(1-\mu \beta)$; but at the same time the distortion voltages for a given fundamental output will be reduced in the same ratio.

Besides reducing the distortion, negative feedback reduces the rate of change of amplifier gain with chang ing supply voltages, reduces some kinds of noise which originate in the amplifier, and can be used to flatten, or otherwise modify, the frequency response. It also has the effect of changing the effective impedance at points where the $\beta$ circuit is connected to the amplifier, and has been used in this way to reduce the impedance of a pentode output valve so as to give better loud-speaker damping, and also to increase the input impedance of valves in high-frequency amplifiers.

It is clear, then, that negative feedback has great attractions for amplifier designers, especially as the explanations often given suggest that the application of feedback is quite a straightforward matter. It is only necessary, apparently, to feed back a voltage from the output terminals via an attenuating network to the input terminals, making sure that this voltage is in the 'right phase' to give reduced gain, and all the benefits follow automatically. Unfortunately, attempts to do this often result in the amplifier becoming unstable, particularly if more than a very modest degree of feedback is aimed at or if the effective frequency range of the amplifier extends above a few kilocycles per second. To understand this trouble, and to determine whether or not it can be overcome, a more rigid mathematical treatment than that mentioned earlier is required.

The simple mathematical treatment as usually given assumes-often tacitly-that $\mu$ and $\beta$ are simple numbers, implying that neither the amplifier nor the feedback network cause any change of phase of the signals they transmit. Actually, although this may be approximately true near the middle of the working frequency band, phase-shifts always occur at higher and lower frequencies because changing phase is inseparable from falling gain. If the phase characteristics as well as the gain and attenuation characteristics of the amplifier and feedback network are taken into account, a much more useful and comprehensive statement of the effects of feedback can be obtained. Black's paper deals also with this more complex version of the problem, but the results seem to have received comparatively slight attention.

To find the effects of phase-shift in the amplifier, it is convenient to regard the quantity $\mu$ as a vector, of which the modulus $|\mu|$ represents the voltage gain of the amplifier and the angle represents the phase shift through the amplifier. Similar considerations apply to $\beta$. A signal which passes once through the amplifier and then once through the feedback network suffers a change of magnitude $|\mu \beta|$ times, and the corresponding change of phase is $\phi$, say. $\phi$ does not include the fixed $180^{\circ}$ per valve, which can be disregarded for the present purpose. It will be seen that $\mu \beta=|\mu \beta| \cos \phi+j|\mu \beta| \sin \phi$, where $j^{2}=-1$.

The gain of the amplifier is still changed in the ratio $1 /(1-\mu \beta)$ when feedback is applied, but the quantity $(1-\mu \beta)$ is now to be regarded as a vector. If the modulus of $(1-\mu \beta)$ is called $R$, it will be seen that $R=|1-\mu \beta|$ which is equal to ratio of gains before and after feedback is applied, and it can be shown that $R^{2}=1-2|\mu \beta| \cos \phi+|\mu \beta|^{2}$. This equation represents a family of concentric circles of radii $R$ and centre at the point $(1, \hat{o})$. To interpret this equation a diagram is prepared by drawing a number of circles with radii greater than, equal to, and less than unity, with a common centre at the point ( 1 , ô), using polar co-ordinates.

This simple diagram can be used to predict the change of gain resulting from the application of any degree of feedback to any amplifier under a fixed set of conditions provided that $\mu \beta$ is known completely, that is, provided that both $|\mu \beta|$ and $\phi$ are known. A line of length $|\mu \beta|$ is drawn from the origin at an angle $\phi$, and the position of the end of this line with respect to the family of circles indicates the change of gain due to feedback. Thus if the line ends on the circle of radius 5, it means that the gain will be reduced by five times; if the line ends on the circle of radius 0.25 it means that the gain will be made four times greater; if the line ends on the circle of unit radius the gain will be unaltered when feedback is applied. It will be seen at once that the unit-radius circle is of special importance as the boundary between regions of 'negative' feedback, which reduces the gain, and 'positive' feedback, which increases the gain, and of course the region of positive feedback is restricted to points within the circumference of the unit-radius circle. This represents a considerable modification of the usual state. ment that feedback is negative when $\dot{\phi}$ is in the neighbourhood of $180^{\circ}$ and positive when $\phi$ is small or zero. This statement, while being useful as a rough-and-ready guide, gives no indication of what happens when $\phi$ is, say, about $90^{\circ}$ or $270^{\circ}$; moreover, although it is true that feedback is always negative when $\phi$ lies in the neighbourhood of $180^{\circ}$, or more accurately between $90^{\circ}$ and $270^{\circ}$, feedback is not always positive when $\phi$ is zero. Thus if $\phi=0$ and $|\mu \beta|>2$, the line representing $\mu \beta$ will lie along the reference line and it will end at a point outside the unit-radius circle. From what has been said before, this will correspond to a reduction of gain, that is, the feedback will be negative. This is a somewhat surprising result, and if a physical interpretation is sought, it seems at first sight that in an amplifier in which these conditions obtain the voltage fed back from the output terminals through the $\beta$ circuit to the input terminals is more than twice as large as, and exactly in phase with, the input voltage, so that the net input voltage to the amplifier is apparently more than three times as great as before feedback was applied; yet the amplifier gain is reduced. The true explanation is that the phase of the output voltage is changed by $180^{\circ}$ when feedback is applied, so that the feedback voltage is in opposite phase to the input voltage. This case is, of course, of little practical importance because it is difficult to realize the required conditions of $|\mu \beta|>2$ and $\phi=0$ in a 
stable amplifier. It is of interest, however, because it prepares the way for a complete statement of the conditions for stability in an amplifier with feedback. This statement is embodied in Nyquist's criterion, which can be explained with the help of the circle diagram.

Suppose in the case of an amplifier working under constant conditions with a certain degree of feedback, the value of $\mu \beta$ is known over the whole range of frequencies from zero to infinity. Now if a series of lines representing $\mu \beta$ at various frequencies is drawn on the circle diagram and a smooth curve is drawn joining the ends of these lines, the curve will form a closed loop beginning and ending at the origin, since $\mu \beta=0$ at zero and infinite frequencies (direct current amplifier amplifiers being excluded). Nyquist's criterion, which is based on theoretical considerations, says that if the point $(1, \hat{o})$ lies inside the loop the amplifier will be unstable; if the point lies outside the loop the amplifier will be stable. The loop may have a complicated shape, and could, for example, have a lobe crossing the $\phi=0$ line at two points beyond $(1, \hat{o})$, and it is seen that the case discussed earlier in which $\phi=0$ and $|\mu \beta|>2$ is theoretically possible in a stable amplifier.

As a check on Nyquist's work, Peterson, Kreer and Ware $^{2}$ built an amplifier in which the $\mu \beta$ loop had a lobe of this kind, and it was found to be stable.

In practical amplifiers it is often unnecessary to plot a complete $\mu \beta$ loop, and indeed a single point can sometimes be very useful. Thus, if the frequency at which $\phi=0$ can be found and the value of $|\mu \beta|$ at this frequency is measured or calculated, it can generally be assumed that the amplifier will be stable if $|\mu \beta|<1$ and unstable if $|\mu \beta|>1$, since lobes are unlikely to occur in straightforward amplifiers. If $|\mu \beta|>1$ at $\phi=0$ it is often possible, by ex. amining the loop in this region, to find some way of reducing $|\mu \beta|$ without appreciably affecting the performance of the amplifier in the working band. It must be realized, however, that for a given frequency band the degree of feedback possible in a stable amplifier is a fixed finite quantity which cannot be exceeded no matter what number of complicated phase-correctors and other devices are used. The estimation of this quantity is dealt with in a paper by Bode $^{3}$ which gives a complete method, based primarily on Nyquist's criterion, for the theoretical design of a negative feedback amplifier.

'Black, H. S., "Stabilised Feedback Amplifiers", Bell System Tech. J. (Jan., 1934).

${ }^{2}$ Peterson, Kreer and Ware, "Rcgeneration Theory and Experiment", Bell System Tech. J. (Oct., 1934).

BBode, H. W., "Feedback Amplifler Design", Bell System Tech. J. (July, 1940).

\section{CONICAL REFRACTION}

\section{BY SIDNEY MELMORE \\ Yorkshire Museum}

A $\mathrm{N}$ interesting paper on this subject by $\mathrm{R}$. Potter appeared in the year $1841^{1}$. He "chiefly worked with an eye-lens, or else for micrometer measures with a compound microscope" and his conclusions (which are easily verified) are contained in the following passages: "The luminous ring is seen, and seen perfectly only, when the lens is so placed in its distance from the crystal, that what he [Lloyd] calls the two rays, are, in fact, the two virtual images of the luminous point on the first surface. The position of these virtual images within the crystal is found by the formulxe of geometrical optics, their distence from the second surface, when the incidence is nearly perpendicular, being equal to thickness of the plate."

refractive index

"Whether we draw the eye-lens away from the crystal, or push it nearer, we find similar appearances, and we see that the ring was formed by the intersection of two cones (or more correctly of two series of cones), the one converging so as to have the bright spot, we formerly found on the second surface, for its vertex, and the other diverging, and having the ring, formerly found on the second surface, for its base."

From these quotations it is evident that Potter was well aware of the possibility of extending the Duc de Chaulnes's method to the study of anisotropic media; but he gives no practical details, and the full development of this method is due to Sorby ${ }^{2}$.

There is no reference to conical refraction in Sorby's papers ; but they contain a description and figure of an effect directly due to it, which he left without investigation as a "curious appearance".

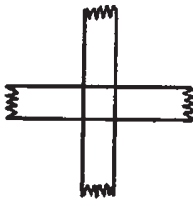

Fig. 1.

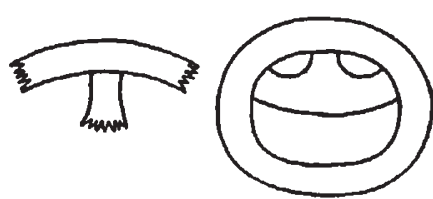

Fig. 2.

Fig. 3.
I observed the effects described below when using a plate of aragonite $8 \cdot 1 \mathrm{~mm}$. thick (cut approximately perpendicular to an optic axis) standing over a pinhole $0.09 \mathrm{~mm}$. in diameter made in a piece of tin foil and viewed under a microscope provided with a 1-in. objective. When the crystal is set so that the refracted rays travel along the acute bisectrix, a cross is seen (Fig. 1); and when viewed through an analyser, first one bar and then the other is extinguished as the analyser is rotated, and the extinction takes place simultaneously at every point in the bar. On tilting the crystal about an axis perpendicular to the optic axial plane, on $\theta$ of the bars gradually becomes curved (Fig. 2). When the tilt amounts to about $6 \frac{1}{2}^{\circ}$ from the acute bisectrix it is apparent that the curved bar is no longer extinguished simultaneously at all its parts, and on closer examination it is seen that the extinguished part of the curve moves through double the angle that the analyser is rotated. By tilting about $8 \frac{1}{2}^{\circ}$ from the bisectrix, the appearance figured by Sorby is seen (Fig. 3). The ring is due to the hollow cone of rays diverging from a focal point considerably out of focus, and the centre of the figure represents the other focal point nearly in focus. The light of each cone is polarized according to the Hamilton-Lloyd law. By altering the focus, the figure changes into an elliptical ring. About $16 \frac{1}{2}^{\circ}$ from the acute bisectrix (that is, in the region of the optic axis), the cones from the two focal points intersect and form a ring approximately circular. In this position the two images of the pinhole lie one above and the other at a nearly equal 